\title{
Rab11a and HSP90 Regulate Recycling of Extracellular $\alpha$-Synuclein
}

\author{
Jun Liu, ${ }^{1,2}$ Jian-Peng Zhang, ${ }^{2}$ Min Shi, ${ }^{2}$ Thomas Quinn, ${ }^{2}$ Joshua Bradner, ${ }^{2}$ Richard Beyer, ${ }^{3}$ Shengdi Chen, ${ }^{1}$ and \\ Jing Zhang ${ }^{2}$ \\ ${ }^{1}$ Department of Neurology and Institute of Neurology, Ruijin Hospital affiliated to Shanghai Jiaotong University School of Medicine, Shanghai, 200025 \\ China, ${ }^{2}$ Department of Pathology, University of Washington School of Medicine, Seattle, Washington 98104, and ${ }^{3}$ Department of Environmental and \\ Occupational Health Sciences, University of Washington, Seattle, Washington 98105
}

Growing evidence suggests that extracellular $\alpha$-synuclein (eSNCA) may play an important role in the pathogenesis of Parkinson's disease (PD) and related synucleinopathies by producing neurotoxicity directly or via activation of glia. However, the mechanisms involved in the trafficking of eSNCA in neurons and/or glia remain unclear. Here, we demonstrated that eSNCA could be resecreted out of neurons via a process modulated by a recycling endosome regulator rab1la in addition to being degraded by an endosome-lysosome system. A quantitative proteomic analysis also revealed numerous proteins through which rab1la might execute its function. One of the candidate proteins, heat shock protein 90 (HSP90), was validated to be interacting with rab11a. Furthermore, geldanamycin, an HSP90 inhibitor, not only prevented resecretion of eSNCA but also attenuated neurotoxicity induced by eSNCA.

Key words: $\alpha$-synuclein; exocytosis; rab11a; HSP90; Parkinson's disease; synucleinopathy; proteomics

\section{Introduction}

Extracellular $\alpha$-synuclein (eSNCA), both monomeric and oligomeric forms, have been proposed as biomarkers of Parkinson's disease (PD) (Borghi et al., 2000; El-Agnaf et al., 2003). eSNCA is also clearly neurotoxic, at least at high concentrations (Sung et al., 2001). Furthermore, eSNCA has been shown to activate microglia (Croisier et al., 2005; Zhang et al., 2005; Su et al., 2008) and astroglia (Klegeris et al., 2006), with subsequent release of proinflammatory factors and cytotoxic reactive oxygen species (ROS) that further facilitate the neurotoxicity of dopaminergic (DAergic) neurons. More recently, in PD patients who received fetal tissue transplantation years ago, Lewy bodies, a pathological hallmark of PD, were found in the transplanted grafts, leading to a provocative hypothesis that host eSNCA may have been transported to the grafts, facilitating the formation of Lewy bodies (Kordower et al., 2008).

Given the essential roles of eSNCA in PD-related mechanisms, there is an urgent need to define the mechanisms by which eSNCA is able to traverse through different cellular compartments. SNCA, originally believed to be only located in the synapses of neurons, has been recently found in both the nucleus and mitochondria (Sangchot et al., 2002). Nonetheless, Lee et al. (2005) have suggested that the majority of eSNCA is derived from cytoplasmic SNCA. After being released, eSNCA can reenter neurons and glia to elicit various biological effects (El-Agnaf et al., 1998;

Received Dec. 31, 2008; accepted Jan. 2, 2009.

This work was supported by grants from the National Institutes of Health (ES012703, NS057567, AG033398, and AG025327) and the National Natural Science Foundation of China (30870879 and 30828031).

Correspondence should be addressed to Jing Zhang, Department of Pathology, University of Washington School of Medicine, HMC Box 359635, 325 9th Avenue, Seattle, WA 98104. E-mail: zhangj@u.washington.edu.

D01:10.1523/JNEUROSCI.6202-08.2009

Copyright $\odot 2009$ Society for Neuroscience $\quad$ 0270-6474/09/291480-06\$15.00/0
Forloni et al., 2000; Zhang et al., 2005), although the mechanisms by which SNCA is released to the extracellular space remain to be characterized.

\section{Materials and Methods}

Reagents. Tissue culture media and supplements were obtained from Invitrogen. All chemical reagents were obtained from Sigma-Aldrich unless otherwise specified. Purified human wild-type recombinant SNCA (MW: $14 \mathrm{kDa}$ ) was obtained from r-Peptide. Anti-lysosome-associated membrane protein (Lamp-1), anti-HSP90, and anti- $\beta$-actin antibodies were obtained from Novus Biologicals. Anti-rablla and anti-human SNCA were provided by Invitrogen. The polyclonal anti-microtubuleassociated protein 2 (MAP2) antibody was provided by Millipore and anti-early endosomal antigen 1 (EEA1) antibody was supplied from Abcam.

Cell cultures. MES23.5 (MES) cells were cultured in DMEM F-12 containing $1 \% \mathrm{~N}-2$ supplement, $2 \%$ fetal bovine serum, and $50 \mathrm{U} / \mathrm{ml}$ penicillin and streptomycin at $37^{\circ} \mathrm{C}$ in a $5 \% \mathrm{CO}_{2}$ humidified incubator.

Condition medium preparation. MES cells were treated with eSNCA at $250 \mathrm{~nm}$ for $3 \mathrm{~h}$, washed extensively with ice-cold PBS to remove bovine serum proteins from culture media, and then incubated in a serum-free medium, before starting chase for different times. The conditioned medium was collected and centrifuged at $1000 \times \mathrm{g}$ for $10 \mathrm{~min}$ at $4^{\circ} \mathrm{C}$, followed by using $0.45 \mu \mathrm{m}$ HPLC syringe filters (Fisher Scientific) to remove cells. Proteins were then precipitated out by $10 \%$ trichloroacetic acid for Western blotting analysis.

Preparation of aggregated eSNCA. Aggregated eSNCA, mainly oligomers, was produced as described by us previously (Liu et al., 2007). A more detailed description and characteristics of the aggregated eSNCA, including size exclusion chromatography and electron microscopic analysis, can be found in the supplemental material (available at www.jneurosci.org).

Immunocytochemistry and immunohistochemistry. Fluorescent labeling of eSNCA was performed with the EZ-Label fluorescein protein labeling kit (Pierce Endogen), and immunostaining was performed as de- 
scribed previously (Zhang et al., 1999; Liu et al., 2007). Images were recorded with a laser-scanning confocal microscope (Bio-Rad LS2000). The procedure for quantifying neurite outgrowth is provided in the supplemental material (available at www.jneurosci.org).

Proteomics and bioinformatics. Details are provided in the supplemental material (available at www.jneurosci.org).

Immunoprecipitation and Western blotting. Tosylated Dynabeads $(1 \times$ $10^{8}$ Dynabeads; Dynal Biotech, Invitrogen) were covalently conjugated to corresponding antibodies according to the manufacturer's instructions. Immunoprecipitation and Western blotting were performed as described previously (Liu et al., 2007).

Rab11a siRNA transfection in MES cells. MES cells were transfected with $10 \mathrm{~nm}$ rat rab1la-specific siRNA [Rn_rab1la_1 HP siRNA (Qiagen); target sequence CGGAATGAGTTTAATCTGGAA located at the 5' terminal of the gene (GenBank accession number NM_031152)]. Constructs or nonspecific control siRNA constructs (Qiagen) were introduced using HiPerFect transfection reagent (Qiagen).

\section{Results}

\section{Secretion of eSNCA by exocytosis}

eSNCA can enter both glia and neurons by endocytosis (Sung et al., 2001; Liu et al., 2007), although the specific mechanisms underlying intracellular trafficking of eSNCA remain to be characterized. In our ongoing investigations, we observed that the amount of internalized eSNCA in MES cells decreased time dependently. Furthermore, in MES cells exposed to preaggregated eSNCA, clearance of the internalized eSNCA followed, at least in part, an endosome-lysosome pathway (supplemental Fig. 1, available at www.jneurosci.org as supplemental material), largely consistent with the report that came out while our manuscript was under preparation, noting that most internalized macromolecules are degraded by lysosomes after trafficking through a series of vesicular compartments (Lee et al., 2008). However, another possibility, which has never been reported previously, is that some of the internalized eSNCA may enter an exocytosis pathway, being secreted out of the cells.

To explore the role of a recycling process in the observed decrease of internalized eSNCA, MES cells were incubated with preaggregated eSNCA for $3 \mathrm{~h}$ and washed extensively with PBS before the amount of eSNCA was determined after different chasing time periods. The results demonstrated that eSNCA (TCAprecipitable) in the medium steadily increased after $6 \mathrm{~h}$ of chasing (Fig. $1 A$ ). In contrast, when the chasing began at a lower temperature $\left(18^{\circ} \mathrm{C}\right)$, a condition known to block vesicular exocytosis, the secretion of eSNCA was inhibited substantially (supplemental Fig. 2, available at www.jneurosci.org as supplemental material). These results clearly suggest that at least a portion of internalized eSNCA was released from the cells through a recycling pathway (exocytosis) in addition to its degradation by an endosome-lysosome pathway (supplemental Fig. 1, available at www. jneurosci.org as supplemental material). Notably, the antibody (Syn211) used here is specific for human SNCA, and is nonreactive with endogenous rodent SNCA in MES cells that are a hybrid of rat mesencephalon and mouse neuroblastoma.

\section{Interaction between rab11a and internalized eSNCA}

Having confirmed that recycling endosomes were involved in the trafficking of eSNCA, we subsequently investigated whether rab11a, an important member of the Rab GTPase protein family that regulates the function of recycling endosomes, participated in endosome-mediated eSNCA exocytosis. To explore whether rab11a interacts with eSNCA, we first investigated whether rab11a colocalizes with internalized eSNCA in neurons. As seen in Figure $1 B$, spatial colocalization of rablla and eSNCA was apparent by confocal analysis after MES cells were treated with eSNCA. This observation was further confirmed with coimmunoprecipitation, using affinity purification with magnetic beads conjugated with either SNCA or rab11a antibodies, followed by immunoblotting with anti-rab11a or anti-SNCA, respectively. The results, shown in Figure $1 C$, demonstrated that rab11a and SNCA were indeed associated with each other, whether SNCA or rab1la was used as the bait initially.

A physical association of two proteins is not necessarily biologically significant. Consequently, we next determined the extent of eSNCA after modulating rab11a levels. Western blotting data, shown in Figure $1 D$, demonstrated that rab1la siRNA not only inhibited the expression of rab11a of MES cells, but also blocked the secretion of eSNCA at 3-6 h of chase (Fig. 1E), arguing that rab11a plays an important role in the resecretion or recycling of eSNCA in MES cells. On the other hand, uptake of eSNCA into the MES cells was not affected when rab11a expression was suppressed with siRNA (supplemental Fig. 3, available at www.jneurosci.org as supplemental material).

\section{Proteomic discovery and validation of the proteins interacting with rab11a}

The data shown in Figure $1 E$ suggest that rab1la is a critical protein regulating the recycling of eSNCA in MES cells. To explore the potential mechanisms by which rab11a mediates this process, the quantitative proteomic profiles in the rabl1a complex were compared between cells treated with vs without eSNCA. A total of 245 proteins were identified that potentially interact with rab1la (supplemental Appendix I, available at www.jneurosci.org as supplemental material); of those, 6 and 12 proteins in three separate experiments increased and decreased, respectively, between MES cells treated with and without eSNCA. More details on the proteomics results and methods used to reduce the dataset and to choose candidate proteins for further validation can be found in supplemental material (available at www.jneurosci.org). A list of proteins that might be important in PD pathogenesis based on current knowledge is provided in supplemental Table 1 (available at www.jneurosci.org as supplemental material). Validation of proteomic results by an alternative means is critical because proteins identified might be incorrect due to the current incomplete protein database. To select candidate proteins for confirmation/validation, the following criteria were used: (1) the identification and quantification of each candidate is based on multiple peptides; (2) BLAST confirms the identification of proteins by unique peptides; (3) there is an antibody commercially available; and (4) its relationship to SNCA has been studied or the proteins belong to a process important in PD but no interaction has been reported with rab11a. With these criteria in mind, HSP90 and HSP70 were selected to confirm their association with rab11a (Zhou et al., 2004; Uryu et al., 2006).

To validate potential protein-protein interactions between rab11a and HSP90 and/or HSP70, coimmunoprecipitation was performed with HSP90, HSP70, or rab11a antibodies individually, and then immunoblotted with anti-rab11a, HSP90, and HSP70, respectively. For HSP70, we were unable to demonstrate its association with rab11a using reciprocal IP (data not shown). In contrast, the results shown in Figure $2 \mathrm{~A}$ demonstrate that HSP90 and rab1la were indeed associated with each other, suggesting a potential protein-protein interaction. One important caveat with the data shown thus far is that the observed interaction may just be a phenomenon occurring in immortalized cell lines, which are significantly different from terminally differentiated neurons in human brains. Thus, the potential interaction 
A

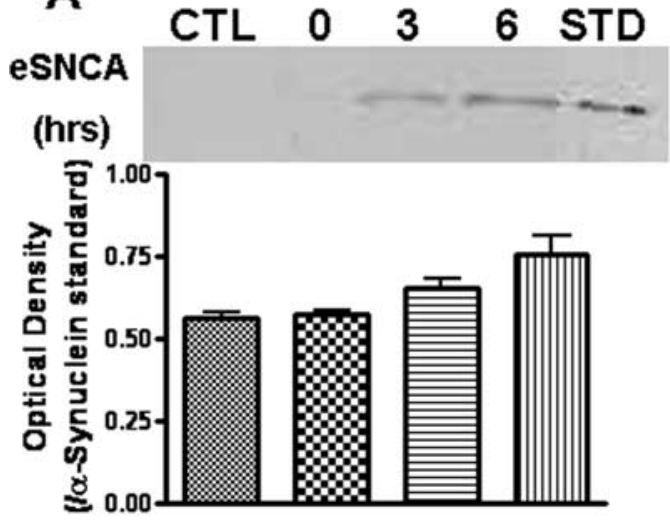

D

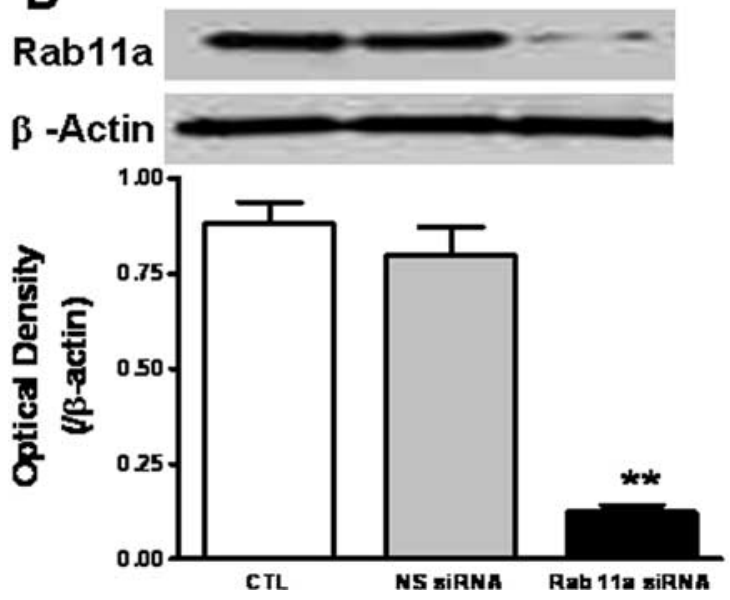

B
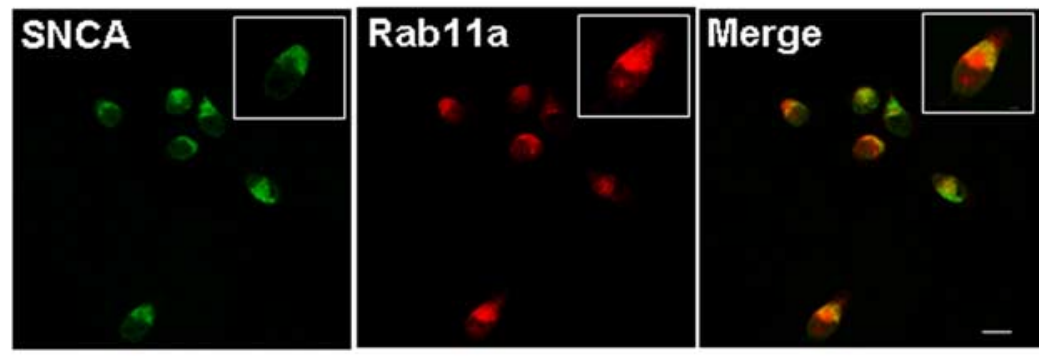

C

Input SNCA-IP CTL

Rab11a

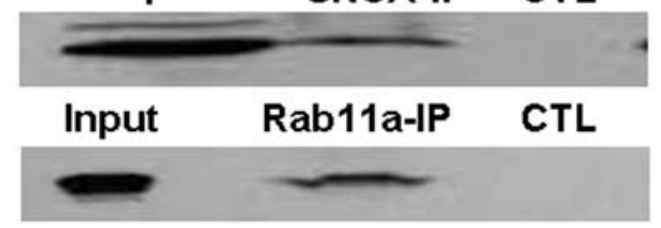

SNCA

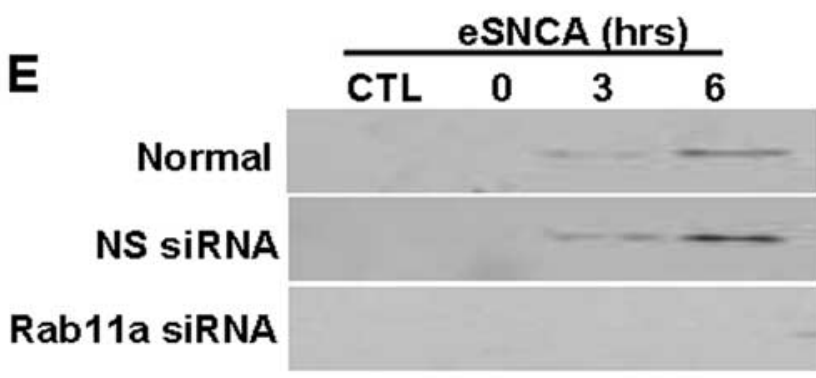

Figure 1. Rab11a and recycling of internalized eSNCA. A, Cells were pulsed with human preaggregated eSNCA for $3 \mathrm{~h}$, washed extensively with PBS followed by adding fresh serum-free medium, and then chased for various time points. At the end of the experiment, the medium was collected and eSNCA was detected at basal (CTL), 3 and $6 \mathrm{~h}$ after the chase, along with standard (STD) SNCA. $\boldsymbol{B}$, MES cells, seeded at $0.05 \times 10^{6} /$ well, were treated with fluorescent labeled eSNCA (green) at $250 \mathrm{~nm}$ for $3 \mathrm{~h}$ before chasing. At an appropriate time, cells were stained with anti-rab11a (red) antibody, followed by examination with confocal microscopy. Note: SNCA was colocalized with rab11a after chase for $3 \mathrm{~h}$ (yellow color seen in the insert of the merged image). $C$, The protein complex of interest was isolated from MES cells homogenate after chasing for $3 \mathrm{~h}$. Coimmunoprecipitation analysis using magnetic beads conjugated with either SNCA (top) or rab11a (bottom) with subsequent pull-down revealed a noticeable protein-protein association between SNCA and rab11a. Input represents original materials. $\boldsymbol{D}$, MES cells were transfected with rab11a siRNA before treatment with eSNCA. Expression levels of rab11a were analyzed by Western blotting with an anti-rab11a antibody $72 \mathrm{~h}$ after gene manipulation, demonstrating that rab11a siRNA effectively inhibited rab11a expression [ ${ }^{* *} p<0.01$, compared with control/nonsense (NS) siRNA groups; $\beta$-actin used as loading control]. $\boldsymbol{E}$, MES cells were transfected with rab11a siRNA before treatment with eSNCA. The amount of eSNCA in condition media was measured by Western blotting analysis with or without rab11a siRNA transfection. A significant blocking in TCA-precipitable eSNCA was found in the condition medium of MES cells transfected with rab11a at 3-6h chasing. Scale bar, $20 \mu \mathrm{m}$.

between rab11a and HSP90 was further investigated in the substantia nigra pars compacta (SNpc) of PD patients with confocal microscopy. The results, shown in Figure $2 B$, revealed that rab11a and HSP90 were indeed colocalized in human tissue, lending further support to a protein-protein interaction between rab1la and HSP90.

Next, we studied the biological role of HSP90 in the recycling of eSNCA by testing the effect of an HSP90 inhibitor geldanamycin (GA) on the resecretion of eSNCA. Figure $2 C$ showed that the resecretion was completely blocked at $3-6 \mathrm{~h}$ of chase following addition of GA. Correspondingly, as predicted, the loss of intracellular eSNCA was attenuated significantly during the pulsechase experiment (Fig. 2D). Together, these results indicate that HSP90 is involved in the cellular routing of internalized eSNCA.

Neurotoxicity induced by eSNCA was rescued by HSP90 inhibitor

As the first step to define the biological roles of HSP90 in the resecretory process of eSNCA, we asked whether an HSP90 in- hibitor could have any effect on the internalized eSNCA in MES cells. In this part of the investigation, MES cells were pretreated with a low level of rotenone (5 nM) or 1-methyl-4phenylpyridinium ion $\left(\mathrm{MPP}^{+} ; 3 \mathrm{~mm}\right)$, the active metabolite of 1-methyl-4-phenyl-1,2,3,6-tetrahydropyridine (MPTP), briefly $(3 \mathrm{~h})$ to stress the system, followed by treatment with eSNCA for 6 h. The results, shown in Figure 3, revealed that rotenone, $\mathrm{MPP}^{+}$, or eSNCA alone did not cause significant loss of neurites, an early indication of neurotoxicity, in MES cells. However, the average length of the neurites was decreased significantly when cells were treated with eSNCA in addition to rotenone or MPP ${ }^{+}$, an effect totally reversed by the HSP90 inhibitor GA. Both rotenone and $\mathrm{MPP}^{+}$are relatively selective mitochondrial complex I inhibitors that increase oxidative stress as well as produce relatively selective DAergic cell death (Przedborski et al., 1996). Thus, the exacerbation of eSNCA-mediated neurite loss by rotenone and $\mathrm{MPP}^{+}$(Fig 3) argues that mitochondrial inhibition, or related processes, predisposes MES cells to neurite loss when treated with 250 nM SNCA. To this end, it should be emphasized 


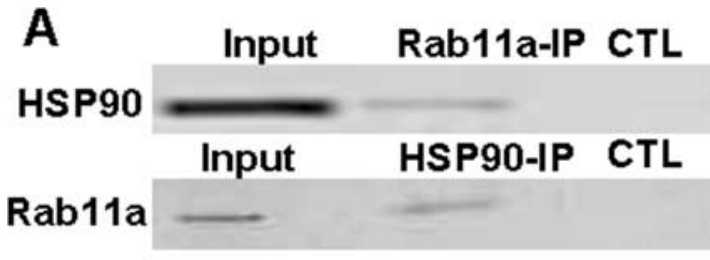

B
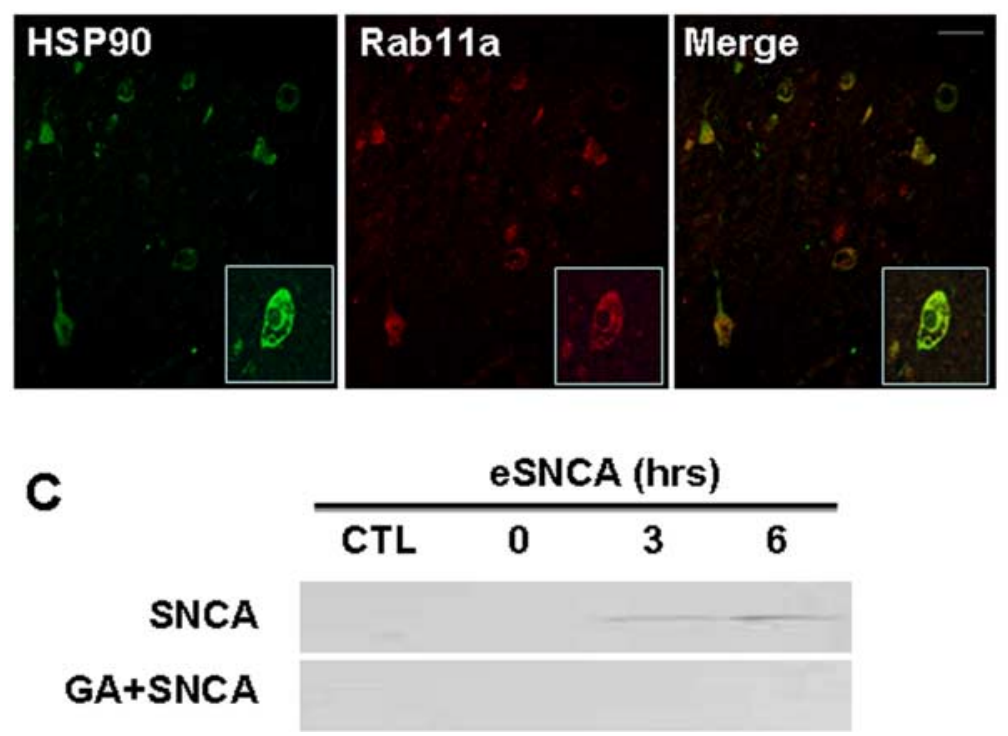

Internalized SNCA (hrs)

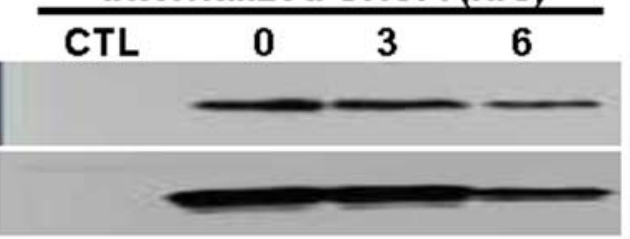

GA+SNCA

$\beta$-Actin

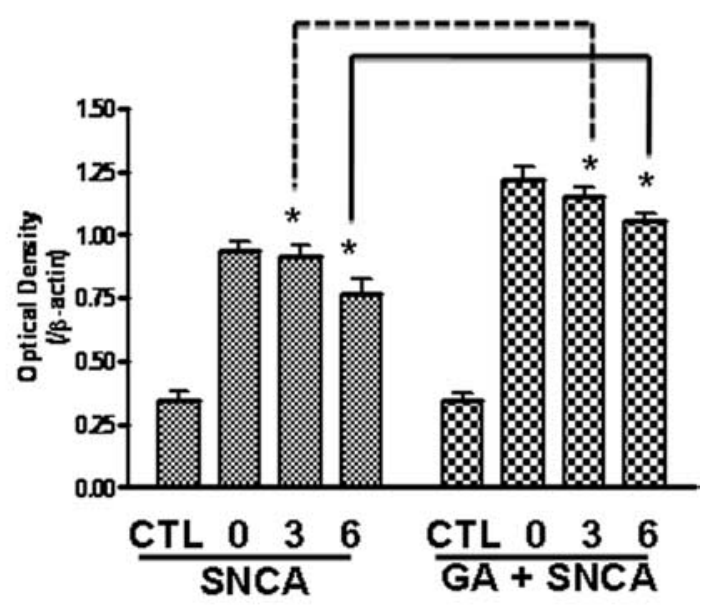

Figure 2. HSP90, rab11a, and the exocytosis of internalized eSNCA. $\boldsymbol{A}$, The protein complex of interest was isolated from MES cells homogenate after eSNCA treatment for $3 \mathrm{~h}$, and detected by anti-HSP90 (top) and anti-rab11a (bottom), respectively. Coimmunoprecipitation analysis using magnetic beads conjugated with either HSP90 or rab11a revealed a clear association between HSP90 and rab11a (Input represents original materials). $\boldsymbol{B}$, The midbrain slides of PD patients were incubated with anti-HSP90 (1:200) or anti-rab11a (1:180), followed by detection with fluorescent labeled anti-mouse (Alexa Fluor 488) and anti-rabbit (Alexa Fluor 568) secondary antibodies, respectively. Colocalization of two proteins is demonstrated as a yellow color shown in the merged confocal image. $C, D, M E S$ cells were pretreated with $1 \mu \mathrm{m}$ GA before the addition of eSNCA for $3 \mathrm{~h}$ before pulse. At the end of the experiment, media and cells were collected, respectively. The amount of extracellular ( $\boldsymbol{C}$ ) and internalized (D) SNCA was measured. Results were obtained from at least three separate experiments. Scale bar, $10 \mu \mathrm{m}$. ${ }^{*} p<0.05$.

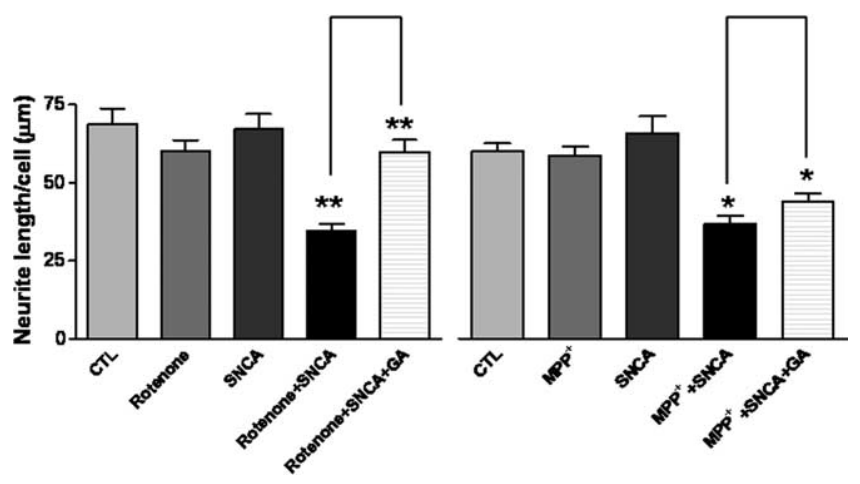

Figure 3. HSP90 inhibitor rescued the loss of neurites induced by eSNCA. MES cells, seeded in four-well chamber slides, and grown to confluency overnight, were incubated with rotenone at $5 \mathrm{~nm}, \mathrm{MPP}^{+}$at $3 \mathrm{~mm}$, or eSNCA at $250 \mathrm{~nm}$ or with a combination (i.e., rotenone plus eSNCA or $\mathrm{MPP}^{+}$plus eSN(A), and with or without pretreatment of GA at $1 \mu \mathrm{m}$ for $3 \mathrm{~h}$. Data are means \pm $S D$ of at least three independent determinations. ${ }^{*} p<0.05{ }^{* * *} p<0.01$.

that the concentration of rotenone used ( $5 \mathrm{~nm}$ ) has been shown in our laboratory to produce mitochondrial inhibition consistently in MES cells when assessed 2-3 d after exposure starts (Zhou et al., 2004). Similarly, the fact that treating MES cells with $\mathrm{MPP}^{+}$at $3 \mathrm{~mm}$ alone did not produce appreciable neurotoxicity is also likely a consequence of timing of assessment, because exposure of
MES cells to $3 \mathrm{mM} \mathrm{MPP}{ }^{+}$for $1-2$ d clearly demonstrated significant neurotoxicity (data not shown).

\section{Discussion}

The current study was centered on eSNCA, whose significant impact on the microenvironment of the brain has been emphasized recently. Several novel findings have emerged from this investigation, i.e., (1) internalized eSNCA was recycled out to the extracellular space in addition to its degradation by an endosome-lysosome system; (2) both rab11a and HSP90 appeared to be involved in the process of exocytosis of internalized eSNCA; and (3) inhibition of HSP90 protected against eSNCA-mediated neurotoxicity while attenuating the exocytosis of internalized eSNCA.

The current investigation represents the first report that internalized eSNCA can be resecreted by a temperature-sensitive and time-dependent exocytosis mechanism. The fact that the treatment of cells with eSNCA alone did not result in any neurotoxicity (Fig. 3) has essentially excluded an experimental artifact, i.e., presence of eSNCA (after eSNCA used to treat the cells was washed away) could be secondary to membrane leakage from dying cells. This observation is significant in that it reveals a novel mechanism by which eSNCA can be derived from [besides being released from dying cells and/or being actively released by neurons (Lee et al., 2005)]. it should also be emphasized that, although the roles of eSNCA remain to be defined further, they are 
readily detectable in human CSF and plasma (Borghi et al., 2000; El-Agnaf et al., 2003), and potently activate microglia and astroglia in addition to inducing neurodegeneration directly (Zhang et al., 2005; Klegeris et al., 2006).

It has been suspected for a while that, because SNCA lacks a signal sequence, it must translocate across membranes via an unconventional pathway before it can be secreted, possibly through vesicular exocytosis (Lee et al., 2005). Hence, a potential role of rab1la in the intracellular trafficking of SNCA is worth noting, as it provides yet another piece of evidence that rab family proteins and SNCA are intimately related. For example, aggregated SNCA can disrupt cellular rab homeostasis at multiple steps (Gitler et al., 2008). Furthermore, overexpression of rab1 has been shown to protect against SNCA accumulation and subsequent DAergic neurodegeneration in animal models of PD (Cooper et al., 2006). Of particular interest, however, is that all reported results related to the rab family proteins and SNCA have been targeted at endogenous SNCA to date. Our present data are the first to show that a part of internalized eSNCA was resecreted to cell medium by recycling endosomes via a process regulated by rab11a. It should be stressed that, although the recycling endosome regulators, e.g., rab11a, are largely docked outside of vesicles, it appears that the rab family proteins may interact with endocytosed cargo proteins directly or indirectly. For example, van IJzendoorn et al. (2002) were the first to show that the polymeric IgA receptor (pIgR), a cargo protein, interacts directly with rab3b, controlling IgA-stimulated transcytosis. Interactions between rab11 and the $\mathrm{Ca}^{2+}$ selective channels TRPV5/6 as well as the $\mathrm{TP} \beta$ receptor have also been recently reported (Thériault et al., 2004). Finally, our confocal investigation (Fig. $1 B$ ) indicated that rab11a and eSNCA did colocalize in intact cells. That said, neither coimmunoprecipitation nor confocal colocalization of two proteins necessarily means a direct interaction between two proteins because they could interact indirectly via a docking $\operatorname{protein}(\mathrm{s})$.

The proteins involved in exocytosis and functions, e.g., cell toxicity and potential contribution to Lewy body formation as mentioned in the introduction, of internalized eSNCA on neurons likely reach beyond just rab11a, as proteomic analysis has revealed many candidates potentially associated or having an interaction with rab11a (supplemental Appendix I, available at www.jneurosci.org as supplemental material). As proteins displaying quantitative changes are less likely to be nonspecific binding proteins, we have deliberately focused on those with significant alterations in their relative abundance (supplemental Table 1, available at www.jneurosci.org as supplemental material) after eSNCA treatment in addition to the selection-criteria discussed above. From this list, HSP70 and HSP90 were chosen as the first set of proteins for further validation of their association with rab11a. However, despite previous investigations about HSP70 and SNCA, we failed to confirm the proteomic interaction between HSP70 and rab11a, using immunoprecipitation techniques. This could mean one of two possibilities: (1) HSP70 was identified incorrectly (due to a database issue as discussed earlier), or (2) technical problems associated with immunoprecipitation studies.

On the other hand, HSP90 was confirmed unequivocally to interact with rab11a (Fig. 2A). HSP90 is the core component of an abundant and ubiquitous cytosolic chaperone machine that uses a variety of cochaperones to regulate the activity of different cellular proteins. An earlier investigation has reported that HSP90 could regulate the release of macromolecules (Hegmans et al., 2004) because an HSP90-containing chaperone system modulates the function of guanine nucleotide dissociation inhibitor $\alpha$ ( $\alpha \mathrm{GDI}$ ) to retrieve prenylated rab3a (Sakisaka et al., 2002). In fact, $\alpha \mathrm{GDI}$ serves as a generic regulator for the recycling of each rab family member, thus, it is possible that HSP90 regulates the function of rab11a. Support for the participation of HSP90 in protein trafficking and $\mathrm{PD}$-related pathogenesis can also be noted in experiments demonstrating that exogenously loaded HSP90 could translocate to early endosomes via receptor-mediated endocytosis (Kurotaki et al., 2007), and that HSP90 chaperones are most prominently and consistently colocalized with SNCA in Lewy bodies or SNCA filaments ultrastructurally (Uryu et al., 2006).

The biological consequence of the regulation of the exocytosis of eSNCA by HSP90 is currently unknown. The fact that the inhibition of HSP90 by GA, a specific HSP90 inhibitor, decreased eSNCA-mediated neurotoxicity (along with decreased exocytosis of eSNCA) could suggest that exocytosis of eSNCA might be detrimental to cell viability. However, these two events may be true but unrelated phenomena, as HSP90 has also been reported to play other roles in cell biology. For example, as a highly conserved protein with chaperone activity, HSP90 interacts with many other proteins and substrates involved in signal transduction and cell cycle control (Picard, 2002).

In summary, this Brief Communication has discovered that internalized eSNCA can be managed by exocytosis in addition to endosome-lysosome-dependent degradation. Additionally, rab11a, a protein known to be important in the exocytosis of macromolecules, appeared to regulate eSNCA exocytosis. Among the candidate proteins interacting with rab11a, HSP90's interaction was validated. Finally, we have provided evidence to suggest that HSP90 is not only critical to neurotoxicity induced by eSNCA but also is involved in the recycling pathway of eSNCA. Further elucidation of the detailed mechanisms by which HSP90 mediates the trafficking of eSNCA will likely provide novel insight into neurodegenerative diseases with synucleinopathies, including PD.

\section{References}

Borghi R, Marchese R, Negro A, Marinelli L, Forloni G, Zaccheo D, Abbruzzese G, Tabaton M (2000) Full length alpha-synuclein is present in cerebrospinal fluid from Parkinson's disease and normal subjects. Neurosci Lett 287:65-67.

Cooper AA, Gitler AD, Cashikar A, Haynes CM, Hill KJ, Bhullar B, Liu K, Xu K, Strathearn KE, Liu F, Cao S, Caldwell KA, Caldwell GA, Marsischky G, Kolodner RD, Labaer J, Rochet JC, Bonini NM, Lindquist S (2006) Alpha-synuclein blocks ER-Golgi traffic and Rab1 rescues neuron loss in Parkinson's models. Science 313:324-328.

Croisier E, Moran LB, Dexter DT, Pearce RK, Graeber MB (2005) Microglial inflammation in the parkinsonian substantia nigra: relationship to alpha-synuclein deposition. J Neuroinflammation 2:14.

El-Agnaf OM, Jakes R, Curran MD, Middleton D, Ingenito R, Bianchi E, Pessi A, Neill D, Wallace A (1998) Aggregates from mutant and wild-type alpha-synuclein proteins and NAC peptide induce apoptotic cell death in human neuroblastoma cells by formation of beta-sheet and amyloid-like filaments. FEBS Lett 440:71-75.

El-Agnaf OM, Salem SA, Paleologou KE, Cooper LJ, Fullwood NJ, Gibson MJ, Curran MD, Court JA, Mann DM, Ikeda S, Cookson MR, Hardy J, Allsop D (2003) Alpha-synuclein implicated in Parkinson's disease is present in extracellular biological fluids, including human plasma. FASEB J 17:1945-1947.

Forloni G, Bertani I, Calella AM, Thaler F, Invernizzi R (2000) Alphasynuclein and Parkinson's disease: selective neurodegenerative effect of alpha-synuclein fragment on dopaminergic neurons in vitro and in vivo. Ann Neurol 47:632-640.

Gitler AD, Bevis BJ, Shorter J, Strathearn KE, Hamamichi S, Su LJ, Caldwell KA, Caldwell GA, Rochet JC, McCaffery JM, Barlowe C, Lindquist S 
(2008) The Parkinson's disease protein alpha-synuclein disrupts cellular Rab homeostasis. Proc Natl Acad Sci USA 105:145-150.

Hegmans JP, Bard MP, Hemmes A, Luider TM, Kleijmeer MJ, Prins JB, Zitvogel L, Burgers SA, Hoogsteden HC, Lambrecht BN (2004) Proteomic analysis of exosomes secreted by human mesothelioma cells. Am J Pathol 164:1807-1815.

Klegeris A, Giasson BI, Zhang H, Maguire J, Pelech S, McGeer PL (2006) Alpha-synuclein and its disease-causing mutants induce ICAM-1 and IL-6 in human astrocytes and astrocytoma cells. FASEB J 20:2000-2008.

Kordower JH, Chu Y, Hauser RA, Freeman TB, Olanow CW (2008) Lewy body-like pathology in long-term embryonic nigral transplants in Parkinson's disease. Nat Med 14:504-506.

Kurotaki T, Tamura Y, Ueda G, Oura J, Kutomi G, Hirohashi Y, Sahara H, Torigoe T, Hiratsuka H, Sunakawa H, Hirata K, Sato N (2007) Efficient cross-presentation by heat shock protein 90 -peptide complex-loaded dendritic cells via an endosomal pathway. J Immunol 179:1803-1813.

Lee HJ, Patel S, Lee SJ (2005) Intravesicular localization and exocytosis of alpha-synuclein and its aggregates. J Neurosci 25:6016-6024.

Lee HJ, Suk JE, Bae EJ, Lee JH, Paik SR, Lee SJ (2008) Assembly-dependent endocytosis and clearance of extracellular alpha-synuclein. Int J Biochem Cell Biol 40:1835-1849.

Liu J, Zhou Y, Wang Y, Fong H, Murray TM, Zhang J (2007) Identification of proteins involved in microglial endocytosis of alpha-synuclein. J Proteome Res 6:3614-3627.

Picard D (2002) Heat-shock protein 90, a chaperone for folding and regulation. Cell Mol Life Sci 59:1640-1648.

Przedborski S, Jackson-Lewis V, Yokoyama R, Shibata T, Dawson VL, Dawson TM (1996) Role of neuronal nitric oxide in 1-methyl-4-phenyl1,2,3,6-tetrahydropyridine (MPTP)-induced dopaminergic neurotoxicity. Proc Natl Acad Sci U S A 93:4565-4571.

Sakisaka T, Meerlo T, Matteson J, Plutner H, Balch WE (2002) RabalphaGDI activity is regulated by a Hsp90 chaperone complex. EMBO J 21:6125-6135.

Sangchot P, Sharma S, Chetsawang B, Porter J, Govitrapong P, Ebadi M
(2002) Deferoxamine attenuates iron-induced oxidative stress and prevents mitochondrial aggregation and alpha-synuclein translocation in SK-N-SH cells in culture. Dev Neurosci 24:143-153.

Su X, Maguire-Zeiss KA, Giuliano R, Prifti L, Venkatesh K, Federoff HJ (2008) Synuclein activates microglia in a model of Parkinson's disease. Neurobiol Aging 29:1690-1701.

Sung JY, Kim J, Paik SR, Park JH, Ahn YS, Chung KC (2001) Induction of neuronal cell death by Rab5A-dependent endocytosis of alpha-synuclein. J Biol Chem 276:27441-27448.

Thériault C, Rochdi MD, Parent JL (2004) Role of the Rab11-associated intracellular pool of receptors formed by constitutive endocytosis of the beta isoform of the thromboxane A2 receptor (TP beta). Biochemistry 43:5600-5607.

Uryu K, Richter-Landsberg C, Welch W, Sun E, Goldbaum O, Norris EH, Pham CT, Yazawa I, Hilburger K, Micsenyi M, Giasson BI, Bonini NM, Lee VM, Trojanowski JQ (2006) Convergence of heat shock protein 90 with ubiquitin in filamentous alpha-synuclein inclusions of alphasynucleinopathies. Am J Pathol 168:947-961.

van IJzendoorn SC, Tuvim MJ, Weimbs T, Dickey BF, Mostov KE (2002) Direct interaction between Rab3b and the polymeric immunoglobulin receptor controls ligand-stimulated transcytosis in epithelial cells. Dev Cell 2:219-228.

Zhang J, Perry G, Smith MA, Robertson D, Olson SJ, Graham DG, Montine TJ (1999) Parkinson's disease is associated with oxidative damage to cytoplasmic DNA and RNA in substantia nigra neurons. Am J Pathol 154:1423-1429.

Zhang W, Wang T, Pei Z, Miller DS, Wu X, Block ML, Wilson B, Zhang W, Zhou Y, Hong JS, Zhang J (2005) Aggregated alpha-synuclein activates microglia: a process leading to disease progression in Parkinson's disease. FASEB J 19:533-542.

Zhou Y, Gu G, Goodlett DR, Zhang T, Pan C, Montine TJ, Montine KS, Aebersold RH, Zhang J (2004) Analysis of alpha-synuclein-associated proteins by quantitative proteomics. J Biol Chem 279:39155-39164. 